\title{
命HAD
}

ISSN-L: 2530-5115

DOI: http://doi.org/10.22585/hospdomic.v3i3.74

\section{La revista Hospital a Domicilio en Latindex}

\section{The journal Hospital a Domicilio in Latindex}

\author{
María Sanz-Lorente ${ }^{1,2}$, Cristina Bojo Canales ${ }^{3}$ \\ 1. Universidad Miguel Hernández, Departamento de Salud Pública e Historia de la Ciencia, Campus San Joan \\ d'Alacant, Alicante, España. \\ 2. Conselleria de Sanidad Universal y Salud Pública, Centro de Salud Pública de Manises, Manises, España. \\ 3. Biblioteca Nacional de Ciencias de la Salud. Instituto de Salud Carlos III, Madrid, España.
}

Correspondencia/Correspondence

María Sanz-Lorente

Universidad Miguel Hernández, Departamento

de Salud Pública e Historia de la Ciencia,

Campus San Joan d'Alacant, Alicante, España.

msanzlor@gmail.com

Recibido/Received

16.05.2019

Aceptado/Accepted

13.06.2019
Conflicto de Intereses/Competing interest

Las autoras declaran la inexistencia de conflicto de interés

\section{Agradecimientos/Acknowledgments}

No declarados

CÓMO CITAR ESTE TRABAJO | HOW TO CITE THIS PAPER

Sanz-Lorente M, Bojo Canales C. La revista Hospital a Domicilio en Latindex. Hosp Domic. 2019;3(3):21723 


\section{RESUMEN}

El presente artículo presenta el sistema de información Latindex como producto de colaboración en el ámbito iberoamericano, así como su cobertura e impacto. Se describe el Catalogo 2.0 como servicio de información cualitativa que está basado en una lista de características diseñadas por un grupo de especialistas para Latindex que trabajó en su confección entre 1999 y 2001. Esta lista permite apreciar el grado de calidad editorial de cada revista, los criterios que cumple y los que no.

Al tiempo, se destaca la participación española, no sólo por su cantidad de producción, sino por el nivel académico de la misma, destacando especialmente las revistas de Humanidades y Ciencias Sociales. De todos modos, y de forma global, se puede observar que si bien aún en minoría en cuanto al número de publicaciones, las revistas científicas españolas del área de las Ciencias de la Salud mostraban un elevado cumplimiento de los parámetros incluidos en la metodología Latindex.

Por último se deja constancia de la aceptación de la revista Hospital a Domicilio, enfatizando en el alto nivel de cumplimiento de las características Latindex.

Palabras clave: Sistemas de Información; Publicaciones Periódicas como Asunto; Indicadores Bibliométricos; Indicadores de Producción Científica.

\section{ABSTRACT}

This article presents the Latindex information system as a collaborative product in the IberoAmerican context, as well its coverage and impact. Catalog 2.0 is described as a qualitative information service that is based on a list of characteristics designed by a group of specialists for Latindex that worked on its preparation between 1999 and 2001. This list allows us to appreciate the degree of editorial quality of each journal, the criteria it fulfills and those that do not.

At the same time, Spanish participation stands out, not only for its quantity of production, but also for its academic level, especially the Humanities and Social Sciences journals. Anyway, and globally, it can be seen that although still in a minority in terms of the number of publications, the Spanish scientific journals in the area of Health Sciences showed a high compliance with the parameters included in the Latindex methodology.

Finally, the acceptance of the Hospital at Home journal is recorded, emphasizing the high level of compliance with Latindex characteristics.

Keywords: Information Systems; Periodicals as Topic; Bibliometric Indicators; Scientific Publication Indicators. 


\section{ANTECEDENTES}

Desde la perspectiva de las revistas científicas, Iberoamérica ha tenido tradicionalmente una presencia marginal en el panorama internacional. Sin embargo, en décadas recientes el conocimiento, difusión, acceso y uso de las publicaciones editadas en la región ha sido favorecido considerablemente, entre otras cosas gracias al surgimiento de diversas iniciativas regionales cooperativas de información, que difunden contenidos sobre las revistas académicas y científicas (1).

Han transcurrido poco más de dos décadas desde que se gestó la idea de contar con un sistema de información sobre las revistas académicas del ámbito Iberoamericano (2). Latindex es producto de la cooperación de una red de instituciones que funcionan de manera coordinada para reunir y diseminar información sobre las publicaciones científicas seriadas producidas en lberoamérica. La idea de creación de Latindex surgió en 1995 en la Universidad Nacional Autónoma de México (UNAM), mediante documento fundacional (3), y se convirtió en una red de cooperación regional a partir de 1997. La misión del sistema fue, y es, difundir, hacer accesible y elevar la calidad las revistas académicas editadas en la región, a través del trabajo compartido (4).

El 17 y 18 de febrero de 1997, se llevó a cabo la reunión de instalación de Latindex como una red de cooperación, a la que asistieron los cuatro países fundadores a través de representantes de las siguientes instituciones: Instituto Brasileiro de Informação em Ciência e Tecnologia (Brasil), Instituto de Información Científica y Tecnológica (Cuba), Instituto Venezolano de Investigaciones Científicas (Venezuela) y el anfitrión y convocante, la Universidad Nacional Autónoma de México. Actualmente, incluye revistas de investigación científica, técnico-profesionales y de divulgación científica y cultural que se editan en América Latina, el Caribe, España y Portugal. Además, ofrece información sobre revistas con contenidos "iberoamericanistas" editadas en cualquier parte del mundo. Las revistas pueden ser impresas o electrónicas, de todas las disciplinas científicas. En esta reunión emanaron los principales acuerdos, entre ellos, el reconocimiento del pleno de la reunión por implementar, desarrollar y difundir las acciones de Latindex sobre la base de la cooperación, incorporando más instituciones de países cooperantes. Con la integración de España y Portugal en 1999, se definió su nombre actual: Sistema Regional de Información para las Revistas Científicas de América Latina, el Caribe, España y Portugal (4).

Así pues, el sistema Latindex es uno de principales esfuerzos cooperativos iberoamericanos creados para dar visibilidad y diseminación a la producción científica publicada en las revistas especializadas de su ámbito geográfico (5).

\section{LATINDEX EN LA ACTUALIDAD}

El sistema, ya en 2015, era referente obligado cuando se trataba de conocer sobre las revistas científicas y académicas de Iberoamérica. Su masa crítica de información lo colocaba como el sistema de información con el mayor número de publicaciones periódicas Iberoamericanas de carácter académico y sus registros de revistas impresas y electrónicas superaban a cualquier otro sistema en cuanto a calidad y cantidad de información (2).

Su funcionamiento se base de la cooperación regional a través de una institución responsable en cada país participante. La coordinación general del sistema, el soporte informático, el desarrollo del sitio web y la construcción y mantenimiento del sistema de ingreso de datos en línea está a cargo de la UNAM.

A día de hoy, año 2019, participan un total de 23 países (6), y el número de revistas por país que han ingresado en el Catálogo pueden consultarse en la figura anexa. 


\section{Catálogo}

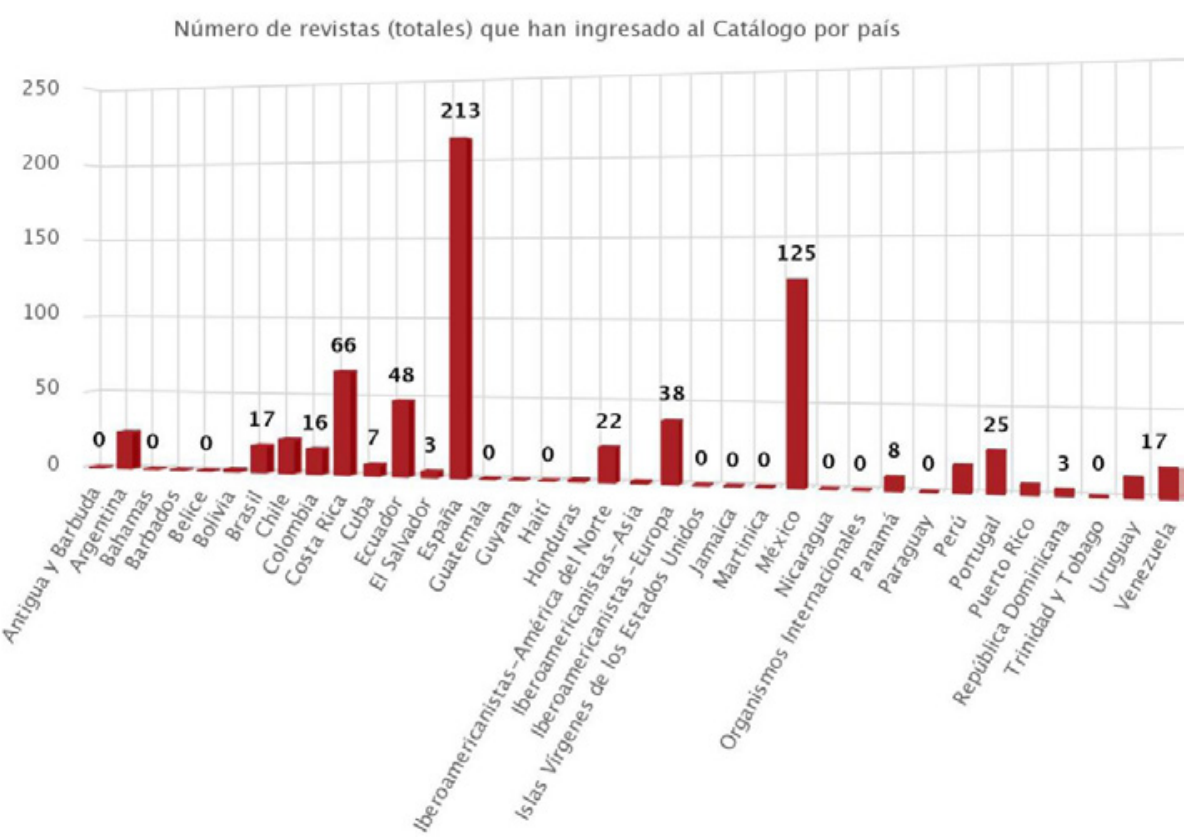

Y, como puede observarse en la figura, su cobertura e impacto han rebasado a la región lberoamericana, al sumarse socios en Europa y de Asia para hacer visibles también las revistas con contenidos latinoamericanistas, hispánicos y lusófonos.

Cuenta con dos servicios principales de información:

1. Directorio: que ofrece datos bibliográficos y de contacto de todas las revistas registradas.

2. Catálogo: compuesto por las revistas con más altos estándares de calidad de acuerdo con la metodología de Latindex.

A diferencia del Directorio, cuya razón de ser es la creación y mantenimiento de una herramienta que recoja y dé cuenta de las revistas existentes en la región (cobertura ahora ampliada a revistas producidas en otras áreas) el Catálogo es un servicio de información cualitativa, de tal modo que únicamente se recogen en el aquellas revistas que cumplen una serie de requisitos mínimos de calidad. Está basado en una lista de características diseñadas por un grupo de especialistas para Latindex que trabajó en su confección entre 1999 y 2001. La lista permite apreciar el grado de calidad editorial de cada revista, los criterios que cumple y los que no (1).

Cada una de las características que conforman el Catálogo 2.0 de Latindex se comprueba única y exclusivamente con la información visible y consultable en la página web institucional u oficial de cada revista; de esta manera se estimula la observancia de normas y buenas prácticas editoriales en la gestión y diseño de las páginas web.

Aparte de los requisitos de postulación, el listado se basa en 38 características ( 7 características básicas, que son todas ellas de obligado cumplimiento, 7 características de presentación, 8 características de gestión y política editorial, 8 características de contenido, 8 características de revistas 
en línea). Estos requisitos y características pueden ser consultados en el sitio "Características de calidad del Catálogo 2.0» (7), dentro de la Web de Latindex.

\section{La participación española en Latindex}

La importancia de España en Latindex queda patente en el estudio realizado por Gantman y Fernández Rodríguez (8), cuyos resultados indicaban que España se destacaba del resto de los países no sólo por su cantidad de producción, sino por el nivel académico de la misma, evaluado mediante la densidad de citas. Este país también exhibe la mayor proporción de artículos de tipo empírico cuantitativo, que se asocia al modelo estadounidense de investigación en la disciplina.

Abejón Peña y Rodríguez-Yunta (9), en 2015, cuantificaron que un 53\% de las revistas españolas del directorio habían ingresado en el catálogo, existiendo un predominio de las ciencias sociales y un crecimiento continuado en el número de títulos.

En España el sistema Latindex ha tenido sin duda un alto impacto que puede valorarse en una triple vertiente (9):

1. Impacto sobre la calidad y visibilidad de las publicaciones, ya Latindex ha sido, ante todo, un modelo que los editores han tomado como referencia para revisar y mejorar sus publicaciones.

2. Impacto sobre los criterios de selección de revistas aplicados en bibliotecas, hemerotecas digitales y bases de datos bibliográficas. Cabe reseñar que la iniciativa Latindex ha sido también un punto de referencia para la selección de revistas, valorado muy positivamente, por parte de las bibliotecas universitarias y de centros de investigación en España. También, hay que referir, que las características editoriales definidas por Latindex se aplicaron también en la puesta en marcha en enero de 2004 del portal de revistas electrónicas de acceso abierto e-revist@s (esta plataforma estableció como elementos de cumplimiento obligatorio un total de 23 de los criterios Latindex) (10).

3. Impacto sobre los sistemas de evaluación científica, al haber contribuido al impulso de las investigaciones para establecer sistemas de evaluación de revistas, que puedan completar o competir con los sistemas basados exclusivamente en las citas recibidas. Ahora bien, este impacto ha afectado, en especial a las revistas de Humanidades y Ciencias Sociales.

De todos modos, y de forma global, se puede observar que si bien aún en minoría en cuanto al número de publicaciones, las revistas científicas españolas del área de las Ciencias de la Salud mostraban un elevado cumplimiento de los parámetros incluidos en la metodología Latindex (11).

\section{La revista Hospital a Domicilio en el Catalogo 2.0}

Desde el inicio de su andadura, el comité editorial de esta revista tuvo especial interés en seguir los indicadores de calidad estipulados para las revistas de las ciencias de la salud y seguir los principales indicadores métricos que avalaran su seguimiento y cumplimiento (12) y, en tal sentido, ha participado en aquellas reuniones que pudieran ser beneficiosas para la difusión y visibilidad de la revista (13).

A inicios del año 2019 el comité editorial de la revista Hospital a Domicilio solicitó la evaluación de la revista Hospital a Domicilio con el fin de ser incluida en el Directorio de Latindex y, en caso de cumplir las características que determinan los estándares de calidad del sistema, su inclusión en el Catálogo.

El pasado día 6 de febrero de 2019 el comité editorial de la revista recibió, vía correo electrónico, la excelente noticia que le comunicaba que la revista había sido evaluada y que había sido regis- 
trada con el folio 27350 (14), cumpliendo en esa fecha 34 criterios de los 38 posibles, ver imagen adjunta. Para ingresar al Catálogo la revista debe cumplir las ocho características obligatorias y al menos 17 de las restantes características, para un mínimo de 25 cumplidas

\section{Editor revista HAD}

\section{De: \\ Enviado el: \\ Para:}

Asunto:

Estimado Comité Editorial:

Pongo en su conocimiento que su revista Hospital a domicilio ya ha sido dada de alta y evaluada según los criterios de Latindex.

Ha sido registrada con el folio 27350 http://www.latindex.org/latindex/ficha?folio=27350 y cumple 34 de los 38 posibles.

Un saludo afectuoso

Esta aceptación supone un claro reconocimiento a la calidad de esta joven publicación y sin duda es un acicate y argumento para invitar a quiénes desarrollan su actividad en el ámbito del cuidado domiciliario a publicar sus resultados de la investigación en esta revista.

En definitiva, y de acuerdo con lo publicado por Alonso y Aleixandre (15), conseguir la inclusión de la revista en una base de datos bibliográfica internacional es un ejercicio de responsabilidad con sus lectores y sus autores que esperan que la revista que leen o en la que publican sus trabajos esté reconocida en los más amplios ámbitos nacionales e internacionales de difusión de la ciencia. Es necesario insistir aquí en que lograrlo no es fruto de la casualidad, sino del buen hacer editorial, de su competencia y rigor.

El hecho de que haya sido indizada implica el reconocimiento científico de la revista y una mayor visibilidad a nivel internacional.

\section{BIBLIOGRAFÍA}

1. Alonso-Gamboa JO, Cetto AM. Latindex: revistas científicas iberoamericanas y cooperación regional. Ci Inf, Brasília. 2015;44(2):197-208.

2. Cetto AM, Alonso-Gamboa JO. Latindex 20 años 1995-2015: Presentación. Ci Inf, Brasília. 2015;44(2):178-Universidad Nacional Autónoma de México (UNAM). Proyecto fundacional (1995): Índice Latinoamericano de publicaciones científicas LATINDEX [página Web]. Latindex: sistema regional de información en línea para revistas científicas de América Latina, el Caribe, España y Portugal. 1995 [citado 25 de abril de 2019]. Disponible en: https://www.latindex.org/ latindex/proyectofund

3. Universidad Nacional Autónoma de México (UNAM). Descripción [página Web]. Latindex: sistema regional de información en línea para revistas científicas de América Latina, el Caribe, España y Portugal. 1997 [citado 25 de abril de 2019]. Disponible en: https://www.latindex.org/ latindex/descripcion 
4. Flores AM, Casado A. Sistema Latindex en Argentina. Ci Inf, Brasília. 2015;44(2):209-Universidad Nacional Autónoma de México (UNAM). Latindex hoy [página Web]. Latindex: sistema regional de información en línea para revistas científicas de América Latina, el Caribe, España y Portugal. 2014 [citado 25 de abril de 2019]. Disponible en: https://www.latindex.org/latindex/hoy

5. Universidad Nacional Autónoma de México (UNAM). Características de calidad del Catálogo 2.0 (Metodología) [página Web]. Latindex: sistema regional de información en línea para revistas científicas de América Latina, el Caribe, España y Portugal. 2019 [citado 25 de abril de 2019]. Disponible en: https://www.latindex.org/latindex/meto2

6. Gantman ER, Fernández Rodríguez CJ. Literatura académica de administración en países de habla hispana: Análisis bibliométrico de la producción en revistas de la base Latindex Catálogo (2000-2010). Investig Bibliotecológica. 2017;31(72):39-DOI: 10.22201/ iibi.0187358xp.2017.72.57823

7. Abejón-Peña T, Rodríguez-Yunta L. La participación española en Latindex: valoración de resultados e impacto sobre la calidad y evaluación de las publicaciones científicas. Ci Inf, Brasília. 2015;44(2):258-Fernández Sánchez, E, Rodríguez Yunta L, Heras JF. La plataforma e-revist@s del portal Tecnociencia. Una experiencia basada en open access. Prof Inform. 14(4):290-Vázquez Valero M, Urdín Caminos C, Román Román A. Las revistas españolas de ciencias de la salud frente a los criterios de calidad editorial Latindex. Rev Esp Doc Cient. 2003;26(4):418-DOI: 10.3989/redc.2003.v26.i4.143

8. Sanz-Valero J, Wanden-Berghe C. Análisis bibliométrico de la producción científica, indizada en MEDLINE, sobre los servicios de salud proporcionados por las unidades de hospitalización a domicilio. Hosp Domic. 2017;1(1):21-DOI: 10.22585/hospdomic.v111.3

9. Grupo de editores de revistas españolas sobre ciencias de la salud (GERECS). Declaración de Sant Joan d'Alacant en defensa del Acceso Abierto a las publicaciones científicas. Hosp Domic. 2018;2(1):5-DOI: 10.22585/hospdomic.v2i1.36

10. Universidad Nacional Autónoma de México (UNAM). Hospital a Domicilio [página Web]. Latindex: sistema regional de información en línea para revistas científicas de América Latina, el Caribe, España y Portugal. 2019 [citado 25 de abril de 2019]. Disponible en: https://www.latindex. org/latindex/ficha?folio=27350

11. Alonso Arroyo A, Aleixandre Benavent R. Importancia para una revista científica de encontrarse incluida en las grandes bases de datos internacionales. Revista de Patología Respiratoria. 2012;15(4):101-DOI: 10.1016/S1576-9895(12)70153-4 